\title{
PATMA Parser of archival tissue microarray
}

\author{
${\text { Lukasz Roszkowiak }{ }^{\text {Corresp., }} 1 \text {, Carlos Lopez }}^{\text {Corresp. } 2}$ \\ 1 Laboratory of Processing Systems of Microscopic Image Information, Nalecz Institute of Biocybernetics and Biomedical Engineering, Polish Academy of \\ Sciences, Warsaw, Poland \\ 2 Molecular Biology \& Research Laboratory (IISPV, URV), Hospital de Tortosa Verge de la Cinta, Tortosa, Spain \\ Corresponding Authors: Lukasz Roszkowiak, Carlos Lopez \\ Email address: Iroszkowiak@ibib.waw.pl, clopezp.ebre.ics@gencat.cat
}

The tissue microarrays are commonly used in modern pathology for cancer tissue evaluation, as it is a very potent technique. Tissue microarray slides are often scanned to perform computer-aided histopathological analysis of the tissue cores. For processing the image, splitting the whole virtual slide into images of individual cores is required. The only way to distinguish cores corresponding to specimens in the tissue microarray is through their arrangement. Unfortunately, distinguishing the correct order of cores is not a trivial task as they are not labelled directly on the slide.

The main aim of this study was to create a procedure capable of automatically finding and extracting cores from archival images of the tissue microarrays. This software supports the work of scientists who want to perform further image processing on single cores. The proposed method is an efficient and fast procedure, working in fully automatic or semi-automatic mode. The $89 \%$ of punches were correctly extracted with automatic selection. With an addition of manual correction, it is possible to fully prepare the whole slide image for extraction in the mean time of 2 min per tissue microarray. The proposed technique requires minimum skill and time to parse big array of cores from tissue microarray whole slide image into individual core images. 


\section{PATMA: Parser of archival tissue microarray}

Lukasz Roszkowiak ${ }^{1 *}$ and Carlos Lopez ${ }^{2^{* *}}$

${ }^{1}$ Nalecz Institute of Biocybernetics and Biomedical Engineering, Ks.

Trojdena 4 Str., Warsaw, Poland

${ }^{2}$ Molecular Biology \& Research Laboratory, Hospital de Tortosa Verge de la

Cinta, IISPV, URV, Tortosa, 43500, Spain

*Corresponding author; Email address: lroszkowiak@ibib.waw.pl, tel:

+48226599143(ext. 124), fax: +48226597030

${ }^{* *}$ Corresponding author; Email address: clopezp.ebre.ics@gencat.cat, tel:

+34977519104 (ext. 2304) 


\begin{abstract}
The tissue microarrays are commonly used in modern pathology for cancer tissue evaluation, as it is a very potent technique. Tissue microarray slides are often scanned to perform computer-aided histopathological analysis of the tissue cores. For processing the image, splitting the whole virtual slide into images of individual cores is required. The only way to distinguish cores corresponding to specimens in the tissue microarray is through their arrangement. Unfortunately, distinguishing the correct order of cores is not a trivial task as they are not labelled directly on the slide.

The main aim of this study was to create a procedure capable of automatically finding and extracting cores from archival images of the tissue microarrays. This software supports the work of scientists who want to perform further image processing on single cores. The proposed method is an efficient and fast procedure, working in fully automatic or semi-automatic mode. The $89 \%$ of punches were correctly extracted with automatic selection. With an addition of manual correction, it is possible to fully prepare the whole slide image for extraction in the mean time of 2 min per tissue microarray. The proposed technique requires minimum skill and time to parse big array of cores from tissue microarray whole slide image into individual core images.
\end{abstract}

\title{
1 Introduction
}

Background In modern pathology, the use of tissue microarrays (TMA) is a common practice [1, 2, 3]. TMA enables researchers to extract small cylinders of tissue, called cores or punches, from histological sections and arrange them in an array on a paraffin block so that multiple samples can be processed simultaneously. From this combined paraffin block slices can be cut and processed like any other histological section. TMA allows a more efficient way to analyse tissue sections, especially when they should undergo further processing, such as staining. It is possible to use immunohistochemistry [4, 5, 6], in situ hybridization (7), or fluorescence in situ hybridization [8] on all cores simultaneously. This way, it lowers the intralaboratory variability of different staining concentrations between slices. Furthermore, it significantly improves processing time, allows obtaining more reproducible results, and greatly reduces the cost of analysis.

TMA are mostly used for cancer tissue evaluation. The analysis of TMA is very similar to regular analysis of histological slides [9] as it is mostly based on visual examination, but instead of one specimen there are many in one slide. Nevertheless, reading big arrays of cores can be burdensome and requires caution. As the cores are not labelled directly on the slide with any text or number, the only way to distinguish specimens is based on their arrangement. The assumed grid structure (array) of the TMA with cores located on the grid nodes is often distorted through the mechanical procedures performed during the glass slide preparation [10, 11]. It is very important to distinguish the correct order that was executed while the TMA was prepared.

Since computer-aided observation and analysis of tissue samples is increasingly common idea and TMA slides can be treated as normal histological slides, it is also possible to make their digital copy. Slide scanners create a virtual image of the whole glass slide [12, 13, 14]. Whole slide images of TMA are enormous, in the context of size, pixels (resolution), as well as disk space. It is impossible to process these images at full resolution due to limited 
memory capabilities of a standard computer workstation. Splitting the whole virtual slide into separate images of individual cores is required for further image processing. The size of these images is suitable for analysis using a 64-bit based computer systems. So the image processing algorithms [15, 16, 17, 18, 19] process cores rather than whole slides.

Although TMA is gaining popularity in clinical practice, it is still more common in research facilities. A vital step to improve the speed and quality of this process is to correctly locate each tissue core in the array. Usually the tissue cores are aligned in a typical microarray, but quite often the TMA cores are askew and incomplete. The problems with automatic selection of cores in TMA include: places where the core should be located, but is actually absent because of technical issues; incomplete cores; cores consisting of many fragments; heavily skewed rows, where cores are relocated in unpredictable manner; stain deposits in the background.

Aim The main aim of this study was to develop a computerized procedure capable of automatically finding and extracting cores from images of TMA. This software was developed on special request from Molecular Biology \& Research Laboratory from Hospital Verge de la Cinta (Institut d'Investigacio Sanitaria Pere Virgili - IISPV), located in Tortosa, Spain. It was named PATMA for Parser of Archival TMA. The procedure is capable of handling core sections under standard conditions - moderately aligned array with well contrasted cores from the background. Once the TMA cores are located, they are stored in separate files for further assessment.

Related works Currently, there is lack of available software that helps the researcher to extract single core image from the whole slide image, which is free of charge, simple to use, and automatic.

There are a few scientific works [20, 21] that can be related to this kind of image processing of TMA. Most of the software focuses on the designing [22] or scoring punches of TMA [23]. Some have been created to support specific study and do not support various data. Other programs apply manual processing [24]. The user must set the number of rows and columns and sometimes select the first core manually. Web-based solutions [25, 26, 27] are not very efficient while working with high-resolution whole slide image, because transferring files with a size of 30 gigabytes or more is rather problematic. Some programs are meant to work with computer-controlled microscope for collecting data [28]; with addition of TMA planning program this may give good results for new research but not while handling archival data. Other solutions found in the literature include Image miner [29], recently published complex computer-aided diagnosis system [30, 31] for TMA, and others [32, 33].

There are also commercial solutions such as TMALab from APERIO or TMADesigner2 from APHELYS. The major disadvantage of commercial software is that it is bound to a specific slide scanner.

\section{Method}

The software was developed in MATLAB R2014b implemented on Intel Core i7-4710MQ @2.50GHz CPU, 16.0GB RAM. The created procedure consists of the steps presented in 
Figure 1: Software procedure

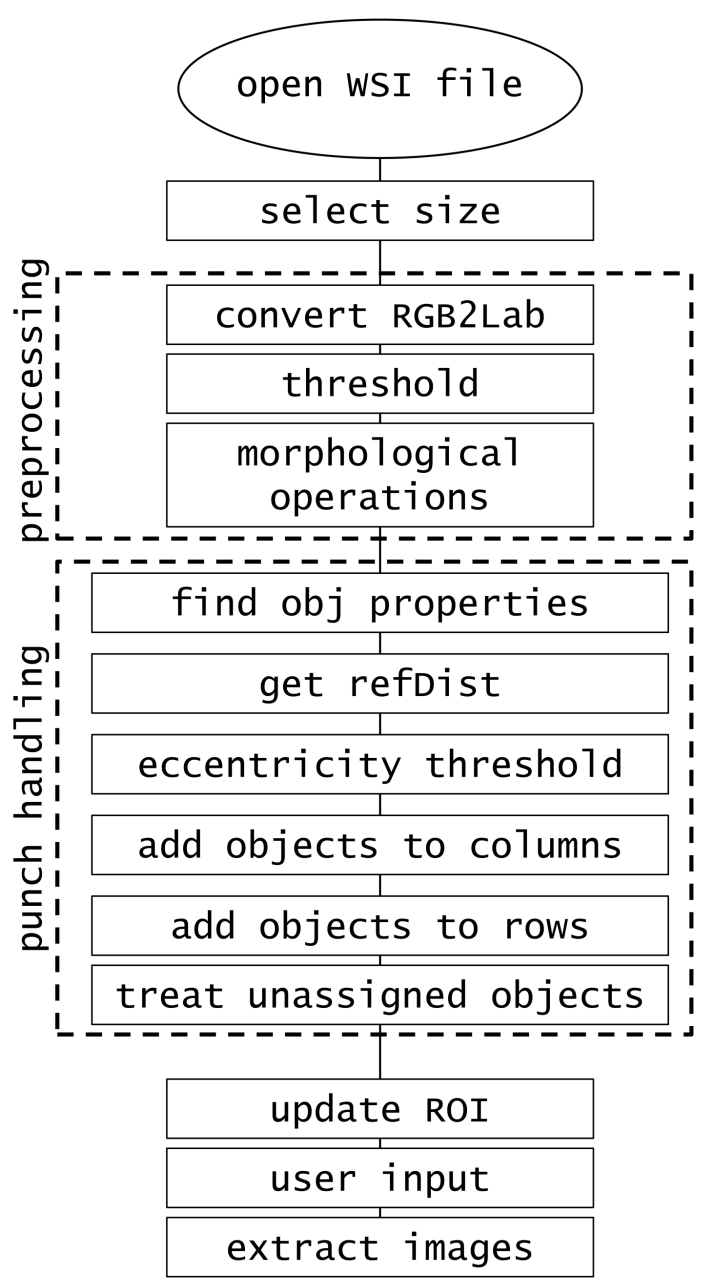

File reading Virtual slide files usually have an inner structure of tiled images that can form the full picture in multiple resolutions. Loaded files are investigated for available resolutions concealed inside. Depending on the user's choice - manual or automatic - working resolution is selected for analysis. Automatic selection always chooses the smallest resolution. Manual selection gives user a choice by providing all available resolutions. The selected image is then preprocessed. As of now the supported format for virtual slides is single-file pyramidal tiled TIFF file format.

Preprocessing The aim of this preprocessing is to create a binary mask with punch location. First, the image is converted from RGB to Lab colour space. Image is binarised with threshold value defined as most common intensity in Luminescence layer (Lab colour space) decreased by 5 . Then, the morphological area opening is executed. Depending on the selected working resolution, objects containing fewer than 1000 pixels are removed from 
images smaller than $10^{7}$ square pixels and objects fewer than 10000 pixels from larger images. Next, a series of other morphological operations including closing, filling, and border clearing are performed (see Algorithm 1). As a result of the preprocessing we obtain black and white images where objects correspond to punch (or fragment of punch) location.

$\overline{\text { Algorithm } 1 \text { Preprocessing procedure with morphological operations description (formulas }}$ according to [34])

1: $I \leftarrow$ input image

2: $\Delta_{s e} \leftarrow$ structuring element

3: procedure PREPROCESS

4: $\quad I_{L}=\operatorname{rgb2lab}(I)$

5: $\quad I_{\text {th }}=$ thresholding $\left(I_{L}\right)$

6: $\quad I_{t h} \circ \Delta_{s e}=\left(I_{t h} \ominus \Delta_{s e}\right) \oplus \Delta_{s e}$

7: $\quad I_{t h} \bullet \Delta_{s e}=\left(I_{t h} \oplus \Delta_{s e}\right) \ominus \Delta_{s e}$

8: $\overline{\overline{I_{t h}}} \Delta_{s e}\left(\partial_{I_{t h}} \cap \overline{I_{t h}}\right)$

9: $\quad I_{t h} \backslash I_{t h} \Delta_{s e}\left(\partial_{I_{t h}} \cap I_{t h}\right)$

$\triangleright$ convert colour space

$\triangleright$ Luminescence layer $\triangleright$ morph opening $\triangleright$ morph closing $\triangleright$ filling holes $\triangleright$ border clear

where: $\ominus$ erosion; $\oplus$ dilation; $\partial$ border mask

Occasionally, strong stain deposit in the background or overstained tissue sections cause extremely unsatisfactory results. In such case, user can choose expert mode and manually modify threshold value as well as size limit of the object to be treated as punch and size of structural element used for morphological operations. If the TMA image was loaded with a wrong orientation, the possibility of rotating the whole TMA is also available in expert mode section. Through this expert preprocessing, a binary map can be previewed by the user to adjust the parameter values to a particular image.

Punch handling We collect properties of found objects (area, centroid, eccentricity, and bounding box - standard MATLAB features). Then, we try to assess the common distance between the cores. This reference distance (refDist) is calculated based on the most circular punch (object with the minimum value of eccentricity) as halved mean of bounding box's width and height. We assume that the distance between the punches is no greater than reference distance. We also assume that punches are objects with eccentricity lower than 0.9. Objects that fulfill the criteria are assigned to columns and rows based on their centroid coordinates (see Algorithm 2 ).

First, objects are assigned to columns one after the other. Among all the unassigned objects we seek the one with the smallest horizontal coordinate. Then, we look for objects with centroid located not further than reference distance, but only in terms of horizontal coordinate. The valid objects are assigned to currently evaluated column. Then, we look for objects in the next column.

After objects are allocated to columns, we try to determine the proper row assignment. First step is similar to that in column search; the object with the lowest vertical coordinate is found. Then, we examine the rest of the objects if they are valid for this row. Objects within the reference distance in terms of vertical coordinate are assigned to currently evaluated row. If this criterion is not met, we have an additional condition based on the centroids of objects 


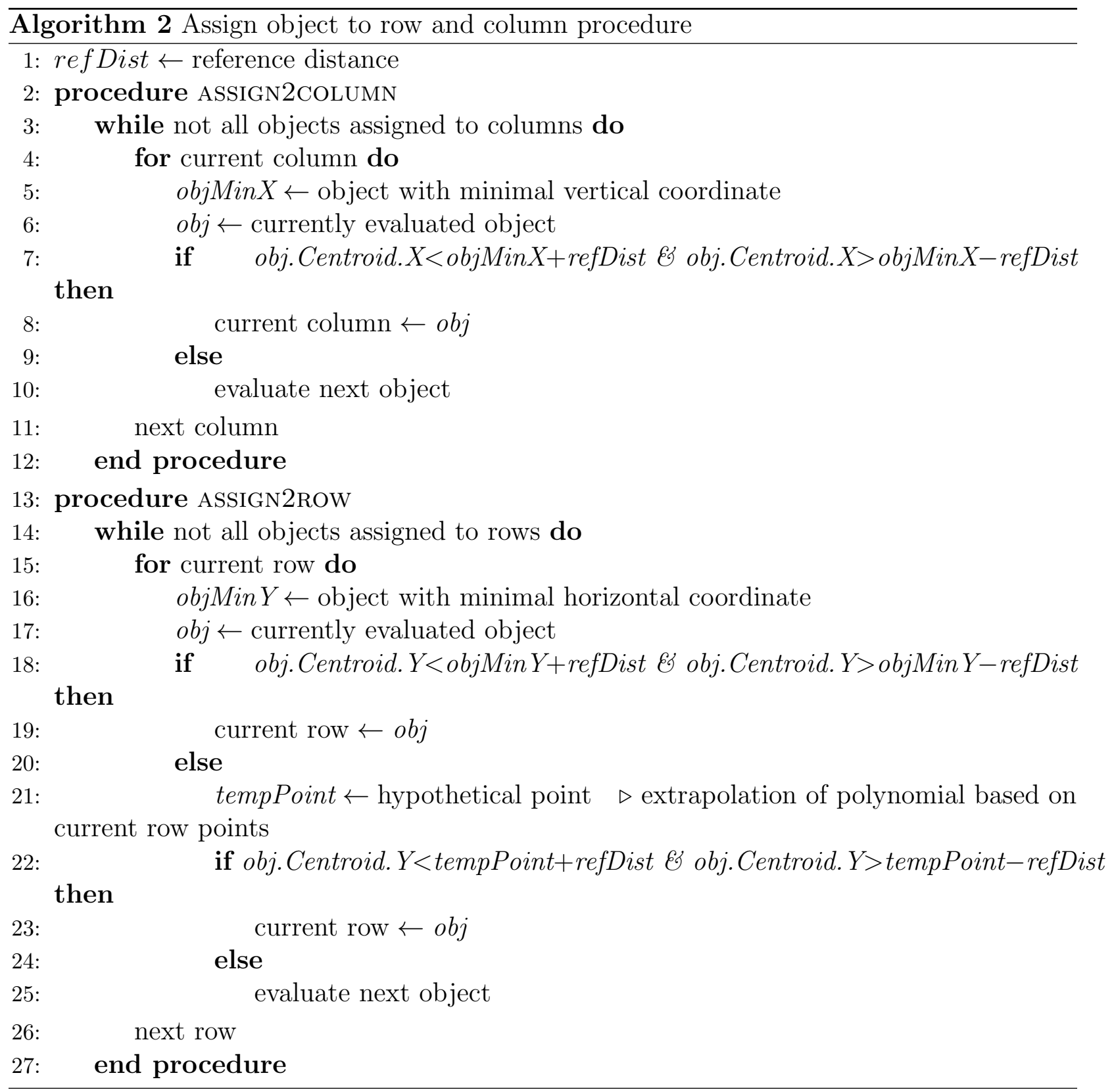


already assigned to the currently examined row. We evaluate the polynomial of second degree based on at least three points. Then, we make an extrapolation of that polynomial with the horizontal value of the currently examined centroid. If the assumed point is within the reference distance of examined centroid, then it is also assigned to the current row. Example of such assignment is shown in Fig. 2.

Figure 2: Example of object assignment to row.
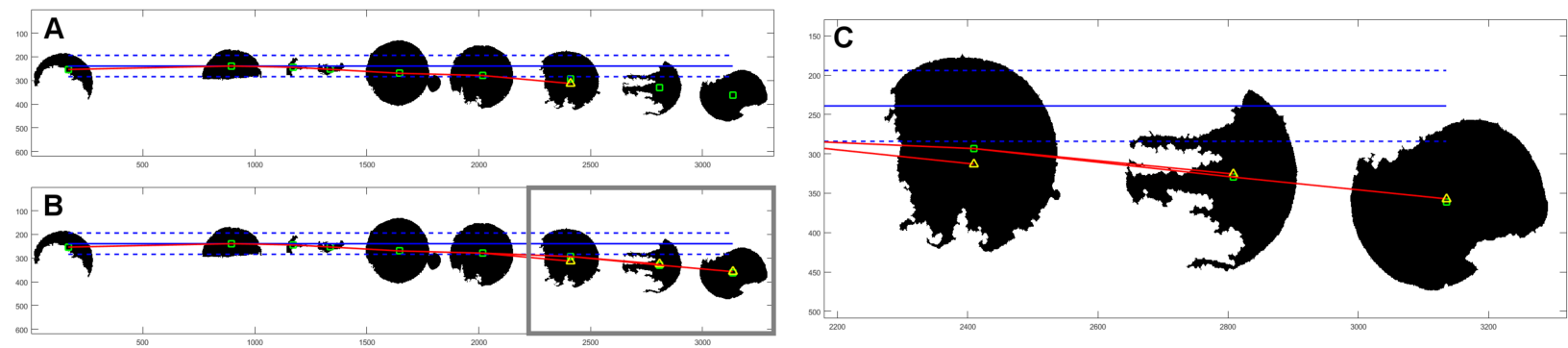

Centroids of objects are marked with green rectangles. Horizontal blue solid line has originated from the centroid of the first object in currently evaluated row. Dashed blue lines mark the reference distance. If the centroid of another object is within the reference distance it is assigned to the currently evaluated row. In this example 6 objects (on the left) meet this term - are within reference distance. Next object's centroid is outside the zone of reference distance so it would be not assigned to this row. In (A) there is a polynominal (red line) of second degree, based on the centroids of already assigned objects, with one extrapolated point (yellow triangle). Extrapolated point has the same horizontal value as the centroid of the next object. Because extrapolated point is closer to the centroid of the object than the reference distance, it is assigned to the currently evaluated row. Image (B) shows final result for this row with three polynominals evaluated for all objects outside of the reference distance from assumed horizontal line. Image (C) is an enlarged fragment of image (B) within gray border.

Then, objects with a missing value of row (or column) are managed. The distance between evaluated object's centroid and the nearest object's centroid is calculated. If it is greater than the reference distance, analyzed object is assigned to the adjacent row (or column). However, for objects closer than the reference distance, closest objects are combined $/ \mathrm{merged}$ into one. Whenever object has no row and column value, a mean of coordinates of each column and row is calculated. Then, this object is assigned to the closest row and column by distance.

Finally, duplicate assignments to rows and columns are treated with the same algorithm. Around every object a region of interest (ROI) similar to the bounding box is created. The regions are adjusted so that there is no overlap. Lastly, correct numerical labels, corresponding to TMA punch numbering, are applied to each ROI.

The designed array structure of the TMA is often distorted, cores can be relocated and may also disappear completely leaving "holes". Because we evaluate assumed reference distance between the cores of TMA, we can figure out where the cores should be in the array despite their actual absence. We assign successive numerical label to the identified "holes" (ROI object with turned off Validity option - see the following paragraph). 
User input The ROI selected in the previous step are presented as an overlay in the processed image, as in Fig. 3. At this stage, the user may apply any necessary corrections. Moving and resizing of selected ROI is possible, because it is draggable. Manual corrections of numerical labels can be applied. Moreover, there is an option of addition and deletion of ROI.

Figure 3: Graphical user interface of the developed software. TMA with overlay of regions of interest presented in the main window. On the right side are subsections related to the file selection, preporcessing, list of ROI, and extraction.

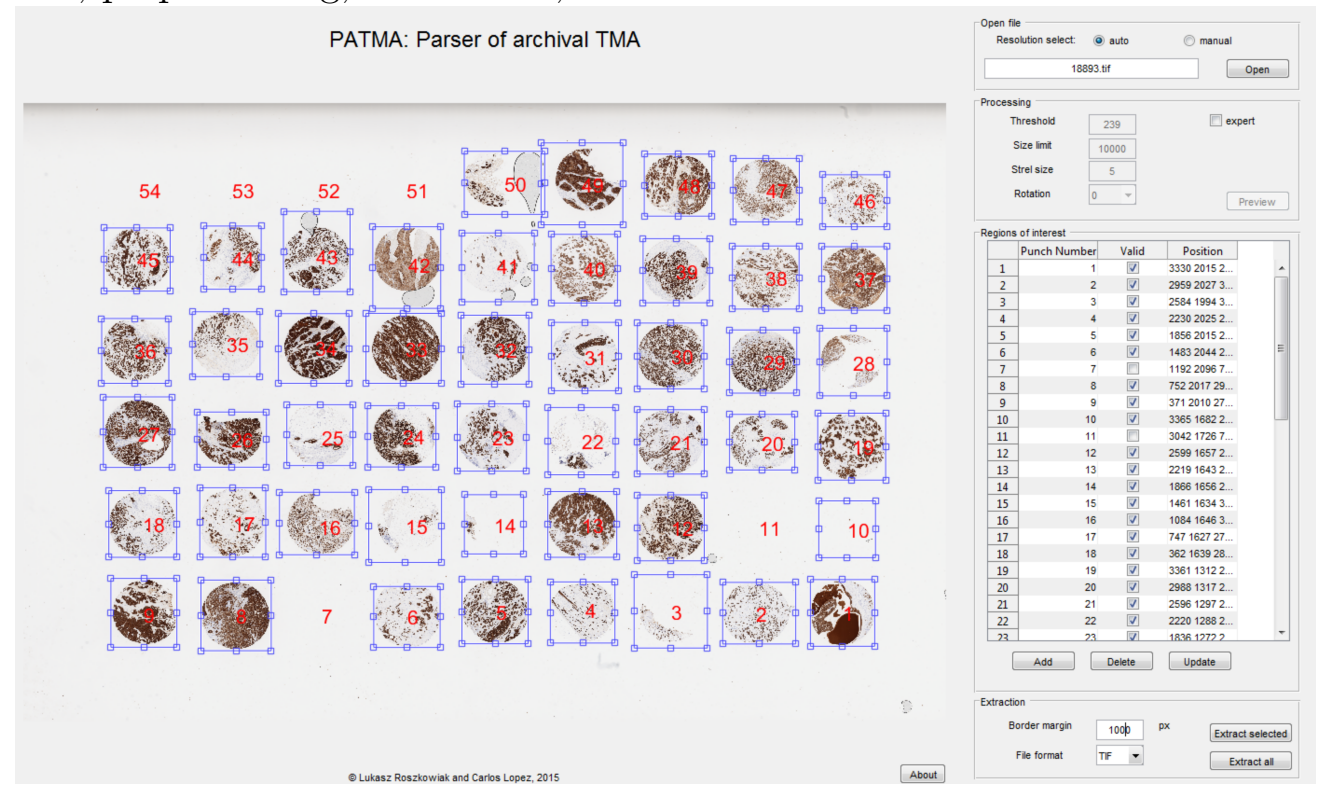

To make editing easier, there is a table presented in the GUI that consists of Numerical label, Position parameters, and Validity. Validity option provides a way to keep correct numbering of punches while there are empty spaces between them. Objects marked as not valid are not extracted in the final step. All of these attributes can be manually changed.

Extraction The selected ROI containing punches are extracted from the maximum available resolution image as separate files. The user can add border margin to the extracted punch to be sure that every pixel with information is inside the selected ROI. Only one punch or all punches from the TMA can be extracted. Images are saved with easily distinguishable filenames in TIFF format.

\section{Results}

There were 27 virtual slides of TMA available to perform the evaluation of this software. The files were received from Molecular Biology \& Research Laboratory from Hospital Verge de la Cinta (IISPV). The glass TMA slides have been prepared according to the procedure described in detail in [6]. The WSI has been acquired using the whole slide scanner (ScaneScope, Aperio) with 40x/0.75 Plan Apo objective lens. We used all the available data 
with the exception of one file that had to be excluded, as the image file contained only a part of the whole TMA which was also rotated. Results are presented in Table 1. In five cases we used resolution chosen manually, as it yielded much better results than automatically selected minimal available resolution (in pyramidal tiled TIFF file). In 11 cases we had to use expert mode (see Preprocessing in the Methodology section) to improve the results, due to strong stain deposit in the background. About $58 \%$ of images were processed by entirely automatic method.

Table 1: All results of core selection on 26 valid cases. Approximate values of segmented miniature image size are presented in Resolution column. Wherever higher resolution was chosen manually, as it yielded much better results than minimal available resolution, there is higher res comment. Punches in TMA are cores planned in the TMA (regardless if the core is still in the image). Selected objects is the number of correctly set ROI (including cores and "holes"). Erroneous objects are all extra, unnecessary ROI set in the image that have to be deleted manually.

\begin{tabular}{|c|c|c|c|c|c|}
\hline Case & Resolution (approx.) & Punches in TMA & Selected object & Erroneous object & Comments \\
\hline 1 & $3800 \times 2700$ & 50 & 50 & 4 & \\
\hline 2 & $4000 \times 2700$ & 50 & 48 & 5 & \\
\hline 3 & $7300 \times 5000$ & 50 & 46 & 1 & higher res \\
\hline 4 & $3800 \times 2700$ & 50 & 44 & 24 & \\
\hline 5 & $3900 \times 2700$ & 50 & 45 & 1 & \\
\hline 6 & $3800 \times 2700$ & 50 & 45 & 40 & \\
\hline 7 & $7800 \times 3900$ & 40 & 38 & 14 & higher res, expert mode \\
\hline 8 & $3800 \times 2000$ & 40 & 38 & 12 & expert mode \\
\hline 9 & $3700 \times 2300$ & 40 & 35 & 12 & expert mode \\
\hline 10 & $3600 \times 1900$ & 40 & 30 & 0 & expert mode \\
\hline 11 & $7300 \times 4500$ & 50 & 45 & 10 & higher res \\
\hline 12 & $2500 \times 1300$ & 50 & 40 & 8 & expert mode, askew \\
\hline 13 & $8700 \times 5200$ & 50 & 47 & 4 & higher res \\
\hline 14 & $7200 \times 5200$ & 50 & 39 & 4 & higher res, askew \\
\hline 15 & $3400 \times 2400$ & 50 & 45 & 29 & \\
\hline 16 & $3500 \times 2400$ & 50 & 48 & 7 & \\
\hline 17 & $3600 \times 2600$ & 50 & 43 & 7 & \\
\hline 18 & $3500 \times 2300$ & 50 & 45 & 7 & \\
\hline 19 & $3500 \times 2300$ & 50 & 49 & 7 & \\
\hline 20 & $3600 \times 2600$ & 50 & 39 & 4 & expert mode \\
\hline 21 & $2000 \times 1300$ & 50 & 41 & 5 & expert mode, askew \\
\hline 22 & $3600 \times 1800$ & 32 & 32 & 6 & \\
\hline 23 & $3600 \times 2300$ & 50 & 45 & 1 & expert mode \\
\hline 24 & $2000 \times 1300$ & 45 & 36 & 4 & expert mode \\
\hline 25 & $3300 \times 1400$ & 32 & 27 & 1 & expert mode \\
\hline \multirow[t]{2}{*}{26} & $3700 \times 2400$ & 50 & 45 & 2 & expert mode \\
\hline & SUM & 1219 & 1085 & 219 & \\
\hline
\end{tabular}

The number of punches per virtual slide varied from 50 to 32. We assume that satisfactory selection consist of ROI with punch. The total percentage of correctly selected cores in 26 evaluated TMA was 89\%. All in all, 1085 out of 1219 punches were selected correctly as a whole core with index of correct row and column. In the majority (16) of cases we achieved $90 \%$ or more correctly selected punches in the TMA slide. In only 7 images PATMA method 
resulted in selecting over 10 erroneous objects. The number of objects which were incorrectly assigned can be most definitely lowered with human interaction (expert mode).

The PATMA's semi-automatic processing approximately takes less than 2 min to fully prepare the TMA slide if the quality of TMA and scanned image is satisfactory. For images that need more human interaction it takes about $5 \mathrm{~min}$.

\section{Discussion}

The processing of fully automatic method is not supervised by the user, hence it may cause unavoidable errors in the extracted images. Eventually, it may lead to additional timeconsuming work (manual task) if punches are extracted with wrong labels (numerical label of punch defines its location in TMA, hence the specimen identification), fragments of punches are extracted separately or punches that are touching border of the image. Thus, we decided to give an option of user supervision in the proposed method.

PATMA identifies not only cores but also "holes" - places where the core should be located, but is actually absent because of technical issues. As a result, we can assign successive numerical labels to all locations of designed array structure of the TMA. It is very useful to identify the "holes" to keep the correct numerical labelling of cores. It is critical to distinguish the correct order that was executed while the TMA was formed as this is the only way to distinguish specimens.

It takes less than 2 min to process a good quality TMA slide image with PATMA and for images that need more human interaction it takes about 5 min. However, complete manual processing is much more laborious and prone to error. Processing the TMA images in full resolution is impossible, for that reason they have to be opened in pieces or the coordinates from their miniatures have to be manually converted to full resolution. It is possible to perform, for example in Fiji (ImageJ), but it would take a lot of effort and time. For the purpose of comparison we performed manual cores extraction on single TMA image with 50 cores and it took about 5 hours.

One of the major problem we encountered are heavily skewed rows of TMA. Relocation of punches often happens during many steps of the TMA slide formation [35, 36. It is impossible to predict if the punches will form askew line or will be randomly relocated. Sometimes two consecutive lines go in different directions in the final image. In this type of deformation it is hard to determine the correct row of the punch even for a human expert. We achieved our goal in case of this problem; from 3 TMA with most askew rows (see Table 1), the $80 \%$ of cores have been correctly selected without human interaction.

Another problem is that the staining of TMA is often not carried out perfectly, which causes deposits and overstained tissue parts in the background of virtual slide [37, 38, 39]. This may result in segmentation of erroneous objects. In 7 images, where PATMA selected over 10 erroneous objects, it was due to stain deposit. Expert mode should be used whenever there is a stain deposit in the background to handle that problem manually.

Incomplete cores or those with many fragments are not a great problem. They usually are well located and merged into one ROI. Artifacts present in the image are much more difficult to handle. Big glue deposit is usually treated as an additional punch or merged with the closest punch. It is a deliberate procedure, because it is easier to delete additional 
ROI than to precisely create a new one. For the slides with extra glue or stain deposits or with cores consisting of many fragments our strategy to remove manually all extra artifacts appears to be efficient. It takes a very small amount of operator's time to make adjustments that whole procedure time takes up to 2 min.

In some cases, the amount of corrections that were necessary may seem high, but they are performed quickly and take much less time than extracting single punches manually. There are 219 erroneous objects (false positives) in evaluated dataset but they can be effortlessly discarded during user inspection. Handling of erroneous object takes no more than 2 seconds. However, 134 (11\%) cores were not found (false negatives) in the evaluated dataset and should be added manually. Handling missing objects consist of adding new ROI, moving it to correct location in the TMA and adjusting the borders. It takes more time and effort than deleting erroneous object but with use of PATMA it is still faster and more straightforward than manual extraction. All in all, it takes $2-5$ minutes to set up all necessary ROI in TMA whole slide image for extraction. In comparison with fully manual processing, which takes hours, the advantage of PATMA is beyond doubt.

Furthermore, we encountered a problem to evaluate our method in comparison to any other related work. None of the related works describe their methodology in detail, hence their solutions (algorithms) are irreproducible. We also tried to contact most of the authors of related works without success. None of the related software was obtainable, as they are only theoretically available on the web. Lastly, the great advantage will be actual availability of the software as we want to share it through MathWorks File Exchange and update it in the future.

\section{Conclusions}

We present free software to efficiently extract separate punches from virtual slides of TMA. It is simple to use for any pathology laboratory, molecular research laboratory, or any other research facility. The great advantages of our software are that it requires none to minimal user input for typical TMA virtual slides and minimum skill and time to operate.

This automation is an advantage for the Molecular Biology \& Research Laboratory compared to the previously used software, which was fully automatic, but worked without considering the location of cores in rows and columns. The time needed to relabel cores, so they would match correct specimen, was considerably longer than the proposed semi-automatic processing. The use of PATMA allows a substantial reduction in the processing time and easier error handling.

Future work In the future, we want to further develop the software to improve its performance. We plan to implement the background elimination algorithm in the preprocessing stage to cope with strong stain deposits. We would like to improve punches' assignment to correct rows and columns, even in heavily skewed tissue microarrays, for example by assuming grid structure that would be matched elastically to actual structure. Finally, we plan to expand the supported file types, so that the other file formats would be handled. 


\section{References}

[1] Olli-P. Kallioniemi, Urs Wagner, Juha Kononen, and Guido Sauter. Tissue microarray technology for high-throughput molecular profiling of cancer. Human Molecular Genetics, 10(7):657-662, 2001.

[2] Mohammad Ilyas, Heike Grabsch, Ian O Ellis, Chris Womack, Robert Brown, Dan Berney, Dean Fennell, Manuel Salto-Tellez, Martin Jenkins, Goran Landberg, Richard Byers, Darren Treanor, David Harrison, Andrew R Green, Graham Ball, and Peter Hamilton. Guidelines and considerations for conducting experiments using tissue microarrays. Histopathology, 62(6):827-839, 2013.

[3] Elton Rexhepaj, Margret Agnarsdottir, Julia Bergman, Per-Henrik Edqvist, Michael Bergqvist, Mathias Uhlen, William M. Gallagher, Emma Lundberg, and Fredrik Ponten. A texture based pattern recognition approach to distinguish melanoma from nonmelanoma cells in histopathological tissue microarray sections. PLoS ONE, 8(5):1-15, 052013.

[4] Urszula Neuman, Anna Korzynska, Carlos Lopez, Marylene Lejeune, Lukasz Roszkowiak, and Ramon Bosch. Equalisation of archival microscopic images from immunohistochemically stained tissue sections. Biocybernetics and Biomedical Engineering, 33(1):63-76, 2013.

[5] Anna Korzynska, Urszula Neuman, Carlos Lopez, Marylene Lejeun, and Ramon Bosch. The method of immunohistochemical images standardization. In Image Processing and Communications Challenges 2, volume 84 of Advances in Intelligent and Soft Computing, pages 213-221. Springer Berlin Heidelberg, 2010.

[6] Carlos Lopez, Cristina Callau, Ramon Bosch, Anna Korzynska, Joaquin Jaen, Marcial Garcia-Rojo, Gloria Bueno, M Teresa Salvado, Tomas Alvaro, Montse Onos, Maria del Milagro Fernandez-Carrobles, Montserrat Llobera, Jordi Baucells, Guifre Orero, and Marylene Lejeune. Development of automated quantification methodologies of immunohistochemical markers to determine patterns of immune response in breast cancer: a retrospective cohort study. BMJ Open, 4(8):1-7, 2014.

[7] Marcial Garcia-Rojo, Gloria Bueno, and Janina Slodkowska. Review of imaging solutions for integrated quantitative immunohistochemistry in the pathology daily practice. Folia Histochemica et Cytobiologica, 47(3):349-354, Jan 2010.

[8] X Du and S Dua. Segmentation of fluorescence microscopy cell images using unsupervised mining. Open Med Inform J, 4:41-49, 2010.

[9] Tomas Seidal, Andre J. Balaton, and Hector Battifora. Interpretation and quantification of immunostains. The American Journal of Surgical Pathology, 25(9):1204-1207, 2001.

[10] Carol B. Fowler, Yan-Gao Man, Shimin Zhang, Timothy J. O'Leary, Jeffrey T. Mason, and Robert E. Cunningham. Tissue Microarrays: Construction and Uses, volume 724 of Methods in Molecular Biology, pages 23-35. Humana Press, Totowa, NJ, 2011. Ed. Al-Mulla, Fahd. 
[11] Martin Werner, Andreas Chott, Alfredo Fabiano, and Hector Battifora. Effect of formalin tissue fixation and processing on immunohistochemistry. The American Journal of Surgical Pathology, 24(7):1016-1019, 2000.

[12] Klaus Kayser, Dominik Radziszowski, Piotr Bzdyl, Rainer Sommer, and Gian Kayser. Towards an automated virtual slide screening: theoretical considerations and practical experiences of automated tissue-based virtual diagnosis to be implemented in the internet. Diagnostic Pathology, 1:1-8, 2006.

[13] Klaus Kayser, Stephan Borkenfeld, Jurgen Gortler, and Gian Kayser. Image standardization in tissue - based diagnosis. Diagnostic Pathology, 5(Suppl 1):S13, 2010.

[14] Liron Pantanowitz, John H. Sinard, Walter H. Henricks, Lisa A. Fatheree, Alexis B. Carter, Lydia Contis, Bruce A. Beckwith, Andrew J. Evans, Avtar Lal, and Anil V. Parwani. Validating whole slide imaging for diagnostic purposes in pathology: Guideline from the college of american pathologists pathology and laboratory quality center. Archives of Pathology \&3 Laboratory Medicine, 137(12):1710-1722, 2013.

[15] R. Redondo, G. Bueno, G. Cristobal, J. Vidal, O. Deniz, M. Garcia-Rojo, C. Murillo, F. Relea, and J. Gonzalez. Quality evaluation of microscopy and scanned histological images for diagnostic purposes. Micron, 43(2 - 3):334-343, 2012.

[16] Lukasz Roszkowiak, Anna Korzynska, Marylene Lejeune, Ramon Bosch, and Carlos Lopez. Proceedings of the 9th International Conference on Computer Recognition Systems CORES 2015, chapter Improvements to Segmentation Method of Stained Lymphoma Tissue Section Images, pages 609-617. Springer International Publishing, 2016.

[17] Tomasz Markiewicz, Stanislaw Osowski, Janusz Patera, and Wojciech Kozlowski. Image processing for accurate cell recognition and count on histologic slides. Analytical and quantitative cytology and histology / the International Academy of Cytology [and] American Society of Cytology, 28(5):281-291, October 2006.

[18] Urszula Neuman, Anna Korzynska, Carlos Lopez, and Marylene Lejeune. Segmentation of stained lymphoma tissue section images. In Ewa Pietka and Jacek Kawa, editors, Information Technologies in Biomedicine, volume 69 of Advances in Intelligent and Soft Computing, pages 101-113. Springer Berlin Heidelberg, 2010.

[19] Anna Korzynska, Lukasz Roszkowiak, Carlos Lopez, Ramon Bosch, Lukasz Witkowski, and Marylene Lejeune. Validation of various adaptive threshold methods of segmentation applied to follicular lymphoma digital images stained with 3,3'diaminobenzidine\&haematoxylin. Diagnostic Pathology, 8(1):48, 2013.

[20] Bernd Lahrmann, Niels Halama, Kathi Westphal, Claudia Ernst, Zeinab Elsawaf, Peter Sinn, Franz X. Bosch, Hartmut Dickhaus, Dirk Jger, Peter Schirmacher, and Niels Grabe. Robust gridding of TMAs after whole-slide imaging using template matching. Cytometry Part A, 77A(12):1169-1176, 2010. 
[21] Rossana Dell'Anna, Francesca Demichelis, Mattia Barbareschi, and Andrea Sboner. An automated procedure to properly handle digital images in large scale tissue microarray experiments. Computer Methods and Programs in Biomedicine, 79(3):197-208, 2005.

[22] Gerhard G. Thallinger, Kerstin Baumgartner, Martin Pirklbauer, Martina Uray, Elke Pauritsch, Gabor Mehes, Charles R. Buck, Kurt Zatloukal, and Zlatko Trajanoski. TAMEE: data management and analysis for tissue microarrays. BMC Bioinformatics, 8(1):1-12, 2007.

[23] T. Amaral, S. J. McKenna, K. Robertson, and A. Thompson. Classification and immunohistochemical scoring of breast tissue microarray spots. IEEE Transactions on Biomedical Engineering, 60(10):2806-2814, Oct 2013.

[24] Wenjin Chen, M. Reiss, and D. J. Foran. A prototype for unsupervised analysis of tissue microarrays for cancer research and diagnostics. IEEE Transactions on Information Technology in Biomedicine, 8(2):89-96, June 2004.

[25] Vincenzo Della Mea, Irene Bin, Maura Pandolfi, and Carla Di Loreto. A web-based system for tissue microarray data management. Diagnostic Pathology, 1(1):1-5, 2006.

[26] F. Demichelis, A. Sboner, M. Barbareschi, and R. Dell'Anna. TMABoost: an integrated system for comprehensive management of tissue microarray data. IEEE Transactions on Information Technology in Biomedicine, 10(1):19-27, Jan 2006.

[27] A. Galizia, F. Viti, A. Orro, D. D’Agostino, I. Merelli, L. Milanesi, and A. Clematis. TMAinspect, an EGEE framework for tissue microarray image handling. In 8th IEEE International Symposium on Cluster Computing and the Grid, 2008. CCGRID '08., pages 741-746, May 2008.

[28] A. Rabinovich, S. Krajewski, M. Krajewska, A. Shabaik, S. M. Hewitt, S. Belongie, J. C. Reed, and J. H. Price. Framework for parsing, visualizing and scoring tissue microarray images. IEEE Transactions on Information Technology in Biomedicine, 10(2):209-219, April 2006.

[29] David J Foran, Lin Yang, Wenjin Chen, Jun Hu, Lauri A Goodell, Michael Reiss, Fusheng Wang, Tahsin Kurc, Tony Pan, Ashish Sharma, and Joel H Saltz. Imageminer: a software system for comparative analysis of tissue microarrays using contentbased image retrieval, high-performance computing, and grid technology. Journal of the American Medical Informatics Association, 18(4):403-415, 2011.

[30] Maria Milagro Fernandez-Carrobles, G. Bueno, O. Deniz, J. Salido, and M. Garcia-Rojo. Automatic handling of tissue microarray cores in high-dimensional microscopy images. IEEE Journal of Biomedical and Health Informatics, 18(3):999-1007, May 2014.

[31] Maria Milagro Fernandez-Carrobles, Gloria Bueno, Oscar Deniz, Jesus Salido, Marcial Garcia-Rojo, and Lucia Gonzalez-Lopez. A CAD system for the acquisition and classification of breast TMA in pathology. Studies in health technology and informatics, 210:756-760, 2015. 
[32] JD Morgan, C Jacobuzio-Donahue, B Razzaque, D Faith, and AM De Marzo. TMAJ: Open source software to manage a tissue microarray database. In Archives of Pathology and Laboratory Medicine, volume 128, page 1094, 2004.

[33] Chih Long Liu, Kelli D Montgomery, Yasodha Natkunam, Robert B West, Torsten O Nielsen, Maggie C U Cheang, Dmitry A Turbin, Robert J Marinelli, Matt van de Rijn, and John P T Higgins. TMA-combiner, a simple software tool to permit analysis of replicate cores on tissue microarrays. Modern Pathology, 18(12):1641-1648, 2005.

[34] Marcin Iwanowski. Metody morfologiczne w przetwarzaniu obrazow cyfrowych [Morphological operations in digital image processing]. Exit, 2009.

[35] Anna Korzynska, Lukasz Roszkowiak, Dorota Pijanowska, Wojciech Kozlowski, and Tomasz Markiewicz. The influence of the microscope lamp filament colour temperature on the process of digital images of histological slides acquisition standardization. Diagn Pathol., 9(Suppl 1):S13, 2014.

[36] Hadi Yaziji and Todd Barry. Diagnostic immunohistochemistry: What can go wrong? Advances in Anatomic Pathology, 13(5):238-246, 2006.

[37] Sara Stromberg, Marcus Gry Bjorklund, Caroline Asplund, Anna Skollermo, Anja Persson, Kenneth Wester, Caroline Kampf, Peter Nilsson, Ann-Catrin Andersson, Mathias Uhlen, Juha Kononen, Fredrik Ponten, and Anna Asplund. A high-throughput strategy for protein profiling in cell microarrays using automated image analysis. Proteomics, $7(13): 2142-2150,2007$.

[38] Marylene Lejeune, Vanessa Gesti, Barbara Tomas, Anna Korzynska, Albert Roso, Cristina Callau, Ramon Bosch, Jordi Baucells, Joaquin Jaen, and Carlos Lopez. A multistep image analysis method to increase automated identification efficiency in immunohistochemical nuclear markers with a high background level. Diagnostic Pathology, $8(1): 1-7,2013$.

[39] Chih Long Liu, Wijan Prapong, Yasodha Natkunam, Ash Alizadeh, Kelli Montgomery, C. Blake Gilks, and Matt van de Rijn. Software tools for high-throughput analysis and archiving of immunohistochemistry staining data obtained with tissue microarrays. The American Journal of Pathology, 161(5):1557-1565, 2002. 